\title{
LA RELIGIÓN EN LA ESFERA PÚBLICA: ANÁLISIS DE LA "CLÁUSULA DE TRADUCCIÓN" DEL DISCURSO RELIGIOSO AL LENGUAJE SECULAR
}

\author{
RELIGION IN THE PUBLIC SPHERE: ANALYSIS OF THE TRANSLATION \\ CLAUSE OF RELIGIOUS SPEECH TO SECULAR LANGUAGE
}

\section{Gonzalo Scivoletto}

\begin{abstract}
RESUMEN
El presente trabajo se ocupa del lugar de la religión en la última etapa de la obra de Habermas. En la primera parte se muestran las diferentes aristas de la cuestión de la religión, poniendo énfasis en los aspectos filosófico-políticos, sobre todo el que concierne a la "traducción" del lenguaje religioso al secular como un "requisito" de acceso a la esfera pública. En la segunda parte, se reconstruye, señalando sus límites o dificultades, el concepto de "traducción" en Habermas. Para ello, se apela, además, a algunas categorías conceptuales propuestas por Jean-Marc Ferry. La traducción en este marco puede ser entendida en dos sentidos: como una reformulación semántica que apunta a ampliar el universo discursivo de origen (traducción como desencapsulamiento) y como una transformación de los géneros pre o para-argumentativos en argumentativos (traducción como cambio de género discursivo).
\end{abstract}

PALABRAS CLAVE: Habermas, religión, postsecularismo, traducción, esfera pública

\begin{abstract}
The present work deals with the place of religion in the last stage of Habermas' work. In the first part the different aspects of the question of religion are shown, emphasizing the philosophical-political aspects, especially the one that concerns the "translation" of the religious language to the secular like a "requisite" of access to the public sphere. In the second part, the concept of "translation" in Habermas is reconstructed, pointing out its limits or difficulties. To this end, it also appeals to certain conceptual categories proposed by Jean-Marc Ferry. The translation in this framework can be understood in two senses: as a semantic reformulation that aims to extend the original discursive universe (translation as "disencapsulation") and as a transformation of the pre or paraargumentative genres into argumentative (translation as discursive genre change).
\end{abstract}

KEYWORDS: Habermas, religion, post-secularism, translation, public sphere 


\section{INTRODUCCIÓN}

En los últimos años, la problemática de la religión en general, y de su rol en el ámbito público en particular, ha despertado un enorme interés académico (más allá de sus campos de estudio tradicionales como la sociología, la filosofía o la antropología de la religión y la teología). Si bien, como sugiere Mendieta, la religión tal vez nunca se fue del todo (Mendieta, 2001), sí es cierto que muchos referentes de la filosofía actual se han ocupado, desde diferentes marcos teóricos y con diferentes objetivos, de la religión ${ }^{1}$. Pero, dentro de los filósofos contemporáneos que se han abocado a la cuestión, quizá uno de los que ha despertado mayor sorpresa ha sido Habermas. Hasta mediados de los '80, la religión no ocupaba un lugar destacado en su obra, aunque por supuesto eso no quiere decir que no tuviera ningún lugar, tal como puso de relieve en su momento Mardones ${ }^{2}$ (1998). Pero dicho lugar era más bien representado por la imagen común de la religión en la tradición de la izquierda hegeliana y de la escuela de Frankfurt, y, de hecho, el mismo Habermas confiesa hacia fines de los 80 que había evitado hablar sobre cuestiones relativas a la teología (Habermas, 1988: 38). Sin embargo, podría decirse que desde su libro El futuro de la naturaleza bumana (Habermas, 2002) y su encuentro con Ratzinger - luego Papa Benedicto XVI-, sus afirmaciones sobre el postsecularismo y el rol de la religión en el ámbito público han provocado una explosión bibliográfica que ha dejado en un segundo plano otros aspectos de la obra de Habermas, incluidos temas como el naturalismo y el determinismo de la libertad, que incluso están fuer-

${ }^{1}$ Esto es particularmente significativo en el "resurgir" de la teología política, ligada a Carl Schmitt, en la tradición filosófica francesa dentro y fuera de la fenomenología (además de Derrida, Nancy, Marion, Henry, Badiou, entre otros), o en la teoría crítica lacaniana de Zizek, por mencionar sólo algunos ejemplos.

${ }^{2}$ La reconstrucción que realiza Mardones sobre la religión en Habermas parte de la tesis de que este entiende a la ética discursiva como la que asume la "función significativa, orientadora e integrativa" de la religión y, si bien reconoce que en el "último Habermas" hay una mayor sensibilidad, su posición representa la línea crítico-ilustrada que remonta a Kant y Marx (Mardones, 1998: 10 ss.). Sin embargo, el posicionamiento de Habermas sobre la religión se modificaría luego de este diagnóstico. Lo que está en discusión desde entonces es cuál es el alcance de ese cambio o "giro religioso". Mi impresión es que hay dos líneas: quienes ponen el acento en la dimensión política o filosófico-política, en el marco de la teoría de la democracia deliberativa, y quienes - muchos menos - lo hacen en la dimensión lingüística y antropológica, en el marco de una reconstrucción genético-evolutiva de la teoría de la acción comunicativa. En ambas líneas, a su vez, pueden encontrarse opiniones enfrentadas respecto al mencionado "cambio". 
temente conectados con ese "giro religioso"3. Tal vez esta preferencia o prioridad se deba al interés social, político y mediático que despierta, sobre todo en Europa, la problemática del terrorismo y la situación de los migrantes y refugiados ${ }^{4}$ — quienes, en muchos casos, desde la perspectiva europea, son vistos como "otros" cuya identidad está fuertemente ligada a la religión, sobre todo al islam-. En América Latina, en cambio, la religión entra en escena, a mi juicio, a través del poder real que tienen todavía determinadas iglesias cristianas - principalmente el catolicismo, pero también iglesias evangélicas — para condicionar algunos procesos políticos, de legislación y gestión pública ${ }^{5}$. Pero más allá de estas consideraciones sobre el papel de las religiones en la actualidad, hay quienes encuentran motivos "más profundos" y menos coyunturales para el retorno de lo religioso, es decir, motivos que no se reducen a un problema político de integración y participación ciudadana o al de poder de lobby de la(s) Iglesia(s). Tales motivos podrían ser descriptos como la ausencia de sentido de una modernidad que ha agotado todas sus fuerzas motivacionales $^{6}$ o la crisis de la creencia en el potencial emancipador de la comunicación y la democracia frente a un capitalismo globalizado e imparable ${ }^{7}$.

${ }^{3}$ De hecho, así lo presenta el propio Habermas en Entre naturalismo y religión, donde habla de "dos tendencias opuestas [que] caracterizan la situación espiritual de nuestra época: la expansión de las imágenes naturalistas del mundo y la creciente influencia política de las ortodoxias religiosas" (Habermas, 2005: 9). Sin embargo, como decía, la atención de la crítica se ha orientado hacia la cuestión eminentemente político-religiosa.

${ }^{4}$ Cuestión que está presente, por supuesto, de manera muy clara en Habermas, como puede apreciarse en esta cita: "Mientras, por ejemplo, una parte considerable de los ciudadanos alemanes de origen turco y credo musulmán viva con mayor intensidad política en la antigua que en la nueva patria, faltarán en la esfera pública y en la urna electoral las voces y los votos correctores que serían necesarios para ampliar la cultura política dominante" (Habermas, 2015: 272).

${ }^{5}$ Un caso muy reciente, es el papel que desempeñó la Iglesia evangélica en la oposición a la paz en Colombia. En Argentina, por ejemplo, si bien el catolicismo ha perdido terreno e importantes "batallas" no ha dejado de ser un factor altamente influyente en los procesos de formación de la voluntad política: en un lapso de treinta años la esfera pública se ha encontrado movilizada por las campañas de la Iglesia Católica en contra de la ampliación de derechos civiles, como la ley de Divorcio (en 1987) o la ley de Matrimonio Igualitario (en 2010), y en la actualidad en contra de la legalización del aborto.

${ }^{6}$ Un ejemplo literario, a mi juicio extraordinario, de este clima de época son las novelas de Michel Houellebecq. En Sumisión, además, esta crisis de Occidente se figura en una potencial islamización de Francia —modelo de la laicidad.

${ }^{7}$ En su ensayo "Lo político: el sentido racional de una cuestionable herencia de la teología política" Habermas lo señala explícitamente: "En los Estados democráticos de bienestar de la segunda mitad del siglo XX, la política tenía todavía la capacidad de dirigir los subsistemas 
No obstante, en este trabajo no me ocuparé principalmente de estas cuestiones relativas al "retorno" o rehabilitación de lo religioso, ni en general ni en la obra de Habermas en particular (sobre todo si los términos del debate se centran en las "reales motivaciones" del autor para un cambio de perspectiva). Sin embargo, sí me interesa aclarar una cuestión general: si bien intento argumentar contra el "prejuicio" acerca de una presunta sabiduría originaria o potencial fuerza normativa vinculante encapsulada en la religión, no por ello pretendo señalar lo contrario, en un tono positivista o secularista a la vieja usanza, esto es, sugerir que la religión es algo arcaico, pura superstición y que debe ser "superada". Creo que es importante mantener, en pro de la rigurosidad, una suerte de "agnosticismo metodológico", pues, si bien es cierto, como dice Habermas, que "no se puede excluir que [las religiones] lleven consigo potenciales semánticos valiosos que desarrollen una energía capaz de inspirar a toda la sociedad una vez que liberen sus contenidos de verdad profanos" (Habermas, 2006: 151), tampoco puede afirmarse que necesariamente haya allí efectivamente un contenido tal de verdad. Sólo mediante un discurso podría determinarse si las afirmaciones religiosas son o no "verdades" y eventual-

divergentes; aún podía contrarrestar las tendencias hacia la desintegración social... Hoy, bajo las condiciones del capitalismo globalizado, las posibilidades de la política para proteger la integración social se están reduciendo peligrosamente”. (Habermas, 2011: 23). ¿Es la religión quien viene a ocupar esa función de integración social? Así lo interpreta López de Lizaga, quien, si bien reconoce que Habermas afirma que la rehabilitación de la religión no se debe a criterios funcionales sino de contenido, afirma: "la integración social mediada por tradiciones religiosas ocupa en sus últimos escritos [de Habermas] el lugar que va desalojando la comunicación tanto en éstos como en la sociedad misma. Con la diferencia, nada irrelevante, de que la comunicación prometía suprimir la dominación, y la religión, en cambio, ya no promete nada, salvo consuelo" (López de Lizaga, 2011: 206).

${ }^{8}$ Habermas aclara explícitamente en qué consiste para él el retorno de lo religioso. Se refiere a "la propagación misionera" de las religiones mundiales, el fundamentalismo y la instrumentalización política del poder de la religión (Habermas, 2015: 265). Sin embargo, en otros lugares varía el tono, y ya no se refiere a estos hechos sociales y políticos, sino a razones más de fondo. Por ejemplo, en un texto sobre la filosofía de la religión de Kant, afirma: "en el Occidente europeo, es una batalla del pasado la autoafirmación ofensiva de la comprensión antropocéntrica de sí y del mundo frente a la comprensión teocéntrica. Así pues, el intento de recuperar en una fe racional ciertos contenidos centrales de la Biblia se ha vuelto más interesante que la lucha contra el engaño clerical y el oscurantismo. La razón pura práctica ya no puede estar tan segura de poder hacer frente a una modernización descarrilada recurriendo únicamente a las intuiciones de una teoría de la justicia [...] Por eso la filosofía de la religión de Kant me interesa desde el punto de vista de cómo es posible apropiarse la herencia semántica de las tradiciones religiosas sin desdibujar el límite entre los universos de la fe y el saber" (Habermas, 2006: 219). 
mente evaluarse su potencial normativo. Ahora, entonces, aunque considero que la religión no es un caso especial y que no tiene por qué haber allí necesariamente "verdades" dispuestas a ser reveladas (así como la "inspiración" motivacional de la religión puede ir en cualquier dirección), creo que este giro hacia la religión permite abrir un problema importante para la filosofía discursiva. Con ello me refiero al problema de la pretensión de comprensibilidad y a las posibilidades o dificultades de "traducir" contenidos semánticos, del cual el discurso religioso es de un tipo más. En ese sentido, retorna y se agudiza el problema de la dependencia del lenguaje secular de la filosofía, con sus raíces religiosas o "culturales" en un sentido amplio, como un problema filosófico y de la filosofía, a saber: su lenguaje. La pregunta que sobrevuela este trabajo, a partir del reconocimiento de la problemática participación de las religiones en el ámbito público, es entonces qué significa que un lenguaje sea secular y si es posible un lenguaje no cosmovisivo - el cual presuntamente debería desempeñar la filosofía.

Por último, no quisiera minimizar, aunque sí matizar, el problema político de la participación de ciudadanos creyentes en la esfera pública. Desde mi punto de vista, el problema filosófico detrás del problema político del rol de la religión en el ámbito público no se reduce a una cuestión de membresía y participación. En primer lugar, por una dificultad de descripción empírica: es factible que por razones históricas, culturales o educativas ciudadanos que no precisamente se reconocen como creyentes esgriman argumentos con contenido religioso o que ciudadanos creyentes "utilicen" un lenguaje presuntamente secular para defender argumentos ya asumidos por convicciones religiosas. El supuesto límite entre ciudadanos seculares y ciudadanos religiosos es sumamente difuso en las sociedades contemporáneas. Como en todo límite, se aprecia desde los extremos, pero entre medio la paleta de colores es bien amplia?. Por eso el problema hoy es, como ha señalado Taylor, no la relación del Estado y la religión, sino la del Estado con la diversidad de posturas básicas (Taylor, 2011: 45) ${ }^{10}$. A mi modo de ver, entonces, para abordar la cuestión de la religión en la esfera pública debemos focalizar en qué tipos de razones son admisibles en el espacio público, que en el fondo es un espacio de razones, más que en los tipos de participantes.

\footnotetext{
${ }^{9}$ Para las dificultades sociológicas de determinar los tipos de creencias, ver Taylor, 2014: 355 ss. ${ }^{10}$ Aun cuando no coincido con Taylor en poner en un mismo nivel doctrinas religiosas con doctrinas filosóficas como el marxismo o el kantismo. En este aspecto, tiene razón Habermas al señalar que el punto de diferenciación es la práctica del culto y la membresía. Ahora bien, sin embargo, a la hora de elaborar razones en un debate las razones no siempre se adscriben total y absolutamente a una doctrina. Volveremos sobre este punto.
} 
En segundo lugar, creo que esta estrategia — contra Habermas - argumentativamente es más coherente — con Habermas-. En efecto, Habermas defiende, por un lado, la completa neutralidad de la esfera formal (las instituciones con poder decisorio como el Parlamento o las magistraturas), mientras que, por otro lado, coloca en la puerta de entrada a la esfera público-política ${ }^{11}$ una "cláusula" de traducción como filtro institucional, "pues sin una traducción lograda no hay ninguna perspectiva de que el contenido de las voces religiosas encuentre acceso a las agendas y negociaciones dentro de las instituciones estatales ni de que "cuente" en el más amplio proceso político" (Habermas, 2006: 140). La pregunta que deberíamos plantear entonces es: ¿qué requisito han de cumplir los argumentos para que cuenten como tales? La respuesta a esta pregunta se resume a que tales argumentos deben estar formulados en un lenguaje públicamente accesible (Habermas, 2006: 142). Si bien Habermas mismo no desarrolla la idea de qué debe entenderse por traducción, cómo realizarla, etc., creo que hay dos direcciones que, al menos, podemos ensayar. En primer lugar, se concibe a la traducción como una especie de "desencapsulamiento", esto es, una potencial ampliación del sentido, destinado originalmente a una comunidad específica, hacia una comunidad "universal". En segundo lugar, se puede concebir a la traducción como un cambio de género discursivo, esto es, del lenguaje pre o para-argumentativo a uno argumentativo. Al final del trabajo examinaré estas dos opciones, entendidas como hipótesis exploratorias y, por lo tanto, no concluyentes.

\section{EL "GIRO RELIGIOSO” DE HABERMAS Y SUS MÚLTIPLES ARISTAS}

Desde mi punto de vista, la religión en la filosofía discursiva del "último" Habermas no sólo se reduce a un problema político o filosófico-político en el marco de la democracia deliberativa. Como decía al comienzo, hay otros aspectos conectados, que tal vez no han recibido tanta atención. Si bien no pretendo desarrollar

\footnotetext{
11 "La neutralidad del Estado en lo tocante a las distintas visiones del mundo no es ciertamente un argumento contra la aceptación de manifestaciones religiosas en el espacio político público, con tal de que los procesos institucionalizados de deliberación y decisión en el plano de los parlamentos, tribunales, ministerios y autoridades administrativas sigan estando claramente separados de la esfera informal de los ciudadanos en la comunicación pública y en la formación de la opinión. La separación «entre Iglesia y Estado» exige que entre estas dos esferas haya un filtro encargado de dejar pasar únicamente las aportaciones "traducidas", esto es, seculares procedentes de toda esa babel de voces del ámbito público trasladándolas hasta las agendas de las instituciones estatales" (Habermas, 2009: 79)
} 
una visión de conjunto y acabada, sino situar el tema que me interesa aquí, a saber, el de la comprensibilidad y la traducción, sí es importante mencionar algunas de esas aristas de la cuestión de la religión.

\section{a) La religión en el espacio público, respuesta a Rawls}

El primer acercamiento es de tipo más bien político y atañe a la forma de participación de las comunidades religiosas en los Estados laicos. En este aspecto, la posición de Habermas, y en general el debate en sí, es el menos denso teóricamente. La solución de Habermas, si bien puede ser discutible en algunos puntos, como se muestra a lo largo de este trabajo, es ciertamente razonable, por lo que creo que la explosión bibliográfica que mencioné antes se debe menos a la cuestión teórica en sí misma que a las posiciones políticas (en un sentido amplio) de los lectores. Así, por ejemplo, quienes defienden el laicismo en una posición más cercana a Rawls, de una fuerte separación de los argumentos religiosos del ámbito público, ven en la posición de Habermas cierta decepción o incluso peligro; del otro lado, aquellos intérpretes más "abiertos” a la religión y la espiritualidad, o ellos mismos creyentes, ven con buenos ojos la sensibilidad de Habermas, en cierto modo, porque se alivia la tensión en ese doble juego creyente-ciudadano, esto es, de quienes velan y viven por determinadas convicciones pero deben morderse la lengua en la esfera pública. En ese sentido, en respuesta a Rawls, Habermas entiende que "el Estado liberal no tiene que transformar la obligada separación entre la religión y la política en una indebida carga mentaly psicológica que no puede ser exigida de sus ciudadanos religiosos" (Habermas, 2006: 137); y a continuación afirma: "por supuesto el Estado tiene que esperar que ellos reconozcan el principio de que el ejercicio de la dominación se ejerce con neutralidad respecto de las visiones del mundo. Todo ciudadano tiene que saber y aceptar que sólo cuentan las razones seculares más allá del umbral institucional que separa a la esfera pública informal de los parlamentos, los tribunales, los ministerios y las administraciones" (Habermas, 2006: 137). Al separar la esfera pública informal de la esfera formal (básicamente, las instituciones del Estado) el proceso de toma de decisiones políticas vinculantes (decretos, leyes, sentencias, fallos, etc.) mantiene su neutralidad respecto de las creencias religiosas, pero el ámbito informal (básicamente la sociedad civil) de conformación de la opinión pública "autoriza" la libre circulación de razones de todo tipo, incluidas las religiosas, incluso en su propio lenguaje si no se ha podido encontrar o elaborar traducciones adecuadas. Esta solución, que tiene a la traducción como bisagra fundamental del proceso de conformación de la voluntad política, evitaría el desdoblamiento de la identidad de los ciudadanos religiosos (Habermas, 2006: 138). 
Frente a esta propuesta de Habermas se han planteado numerosas objeciones, de las que aquí me gustaría mencionar las dos, a mi juicio, más relevantes este trabajo. La primera, se refiere a los puntos de intersección entre las esferas formal e informal (López de Lizaga, 2011). La segunda, a que la corrección de Habermas a Ralws, en cualquier caso, no modifica sustancialmente las cosas, pues los ciudadanos creyentes no pueden participar en tanto creyentes (Lafont, 2009; Prono, 2016). En el primer caso, el problema consistiría en la dificultad de establecer límites en el flujo comunicativo entre la sociedad civil y el Estado. López de Lizaga plantea el hipotético caso de una sociedad civil no secularizada frente a un Estado laico, y se pregunta: “¿Podría [el Estado laico] justificarla [su política] empleando argumentos estrictamente laicos ante una sociedad civil que no estuviera secularizada, y en la que las opiniones de los ciudadanos en torno a asuntos de interés público estuvieran muy influidas por la religión?” (López de Lizaga, 2011: 179). Y más adelante vuelve a preguntarse: “¿podrán [una ley o medida administrativa] alcanzar una base de acuerdo suficiente, que garantice la legitimación de la ley en cuestión, si no disponen de algún lenguaje político común, o de algún conjunto de principios de legitimación de la política independientes de sus respectivas cosmovisiones éticas o religiosas?" (López de Lizaga, 2011: 179. Lo que se reclama aquí es una "base de entendimiento". Según entiendo, esa base de entendimiento sería el sistema de Derechos (esto es, a mi modo de ver, similar a lo que Ferry llama "mediación jurídica"). A estas objeciones creo se podría responder lo siguiente. En primer lugar, es difícil pensar una sociedad totalmente no secularizada y uniforme, es decir, una sociedad totalmente católica, por ejemplo, y además una sociedad tal difícilmente generaría (o hubiese generado) instituciones estatales laicas porque habría una total identificación entre la sociedad civil y el Estado. Si existe el Estado laico, al menos desde un punto de vista histórico, no es porque existan sociedades secularizadas, sino porque existen minorías religiosas. En segundo lugar, si una sociedad fuera muy religiosa, los ciudadanos están "fuertemente influenciados por la religión", difícilmente dejen de responder a ese influjo porque se vean obligados a utilizar otro lenguaje: precisamente esto es lo que sucede con el secularismo estratégico. Por ejemplo, hoy en día las justificaciones de los grupos religiosos de sus posturas anti-aborto apelan al lenguaje de la ciencia ("hay vida humana desde la concepción, desde un punto de vista genético") y al derecho ("si desde el momento de la concepción hay vida humana, entonces el aborto es un crimen"). En este sentido, es preferible que las religiones blanqueen, por así decir, sus verdaderas razones, y que en la esfera informal digan lo que quieran. Para el funcionamiento de una democracia y de un Estado de Derecho es suficiente con que esas opiniones, 
en tanto religiosas, queden fuera de los órganos de decisión del Estado. Pero, dado que interpreto a las religiones, en el esquema general de la comunicación públicopolítica $^{12}$, como grupos de presión (en un nivel similar a las asociaciones, corporaciones o lobbys, etc.) no veo por qué se les debe imponer que no hablen en su propio lenguaje, que intenten instalar determinadas problemáticas en la agenda pública y enmarcarlas en su perspectiva.

A mi modo de ver, el segundo tipo de objeción a la posición de Habermas se conecta con el primero. El núcleo de este argumento, tal como explicita Prono, es que básicamente las razones religiosas son contrarias a la razón, son dogmáticas, y, por lo tanto, un creyente "verdadero" no puede poner en duda sus convicciones (Prono, 2016: 10). Con lo cual, el problema de la traducción, desde esta perspectiva, es superfluo. Los contenidos de la fe no tienen nada que aportar a una democracia deliberativa y un Estado de Derecho que se sustenta sobre sí mismo, lo que le queda a la religión es o adaptar sus convicciones a ese lenguaje o quedarse relegada a su propio ámbito: los ciudadanos religiosos o, mejor dicho, sus razones, no pueden participar en tanto que religiosas.

Ahora bien, sin objetar la objeción, sí quisiera señalar un supuesto, creo, problemático. Este planteo presupone que ya sabemos qué es un argumento religioso y qué no, y supone dos entidades claramente delimitables entre sí, creyentes y no creyentes. Volveré sobre este punto más adelante, cuando me refiera estrictamente a la traducción. Pero lo que me gustaría señalar por ahora es que si se ponen filtros demasiado rígidos se corre el riesgo de censura y de exclusión, y este es un problema político mucho más serio a largo plazo. Pero, además, también hay un problema pragmático que, hasta donde he podido observar, no suele señalarse. En la esfera formal, las instituciones del Estado, el discurso suele estar institucionalizado, con protocolos y delimitación de funciones, entre ellas, la de los moderadores, quienes deben velar por el cumplimiento de tales protocolos o procedimientos. En el caso de una discusión parlamentaria o judicial, por ejemplo, existen mecanismos muy estrictos de resguardo de la neutralidad de las propias instituciones, de acusación y apelación. En cambio, en la esfera público-política o débil, no existen ni pueden existir tales mecanismos, excepto en casos de estricta gravedad. Pero tampoco es conveniente una excesiva judialización de la comunicación pública, la cual por principio es relativamente espontánea. Si bien comparto el escepticismo

\footnotetext{
${ }^{12}$ Me refiero al diseño de la comunicación pública de masas propuesto por Habermas en “'Tiene aún la democracia una dimensión epistémica?" (Habermas, 2009: 158 ss.). (Ver, sobre todo, los diagramas 1 y 2).
} 
respecto de la genuina transformación de las convicciones religiosas en argumentos públicos, no me atrevo a afirmar, sin embargo, que todas las convicciones religiosas sean dogmáticas y que la única posibilidad para un creyente sea "disfrazar" su discurso. Por eso planteaba que la solución de Habermas era "razonable": neutralidad del Estado y una sociedad civil permeable y fluida, aún con los riesgos que eso conlleve. Ahora bien, creo que es importante distinguir la dimensión política, que acabo de describir, de la presunta "situación privilegiada" de la religión, en cuanto a su capacidad motivacional y como recurso de sentido.

\section{b) La religión como recurso de sentido y motivación}

Otro aspecto es, entonces, la valoración que se realiza sobre la religión, lo cual es muy diferente al análisis sobre su lugar en un modelo como la democracia deliberativa o incluso de la teoría de la acción comunicativa. Aquí se puede encontrar en los textos de Habermas una actitud por momentos condescendiente, que lleva a exagerar ciertos rasgos en detrimento de otros. Con lo cual, frente a la actitud "excesivamente" secularista ${ }^{13}$ habría llegado el momento, en estas sociedades postseculares, de cambiar la actitud beligerante y reconocer la potencialidad del discurso religioso. Pero, una cosa es cambiar la actitud "combativa" contra la religión, y otra muy distinta es atribuirle algún tipo de "privilegio" cognitivo. En el siguiente fragmento se puede ver cómo se solapan, a mi juicio, estas dos cuestiones. Dice Habermas: "El Estado no puede desalentar a los creyentes y a las comunidades religiosas para que se abstengan de manifestarse como tales también de una manera política, pues no puede saber si, en caso contrario, la sociedad secular no se estaría desconectando y privando de importantes reservas para la creación de sentido" (Habermas, 2006: 138). En primer lugar, hay que decir que el Estado no debería desalentar a nadie, en la medida que se conciba, como lo hace la democracia deliberativa y la ética discursiva, la esfera pública como la institucionalización del discurso argumentativo. Nadie puede ser legítimamente excluido y todos los afectados/interesados deben poder expresar sus razones. Ahora bien, y en esto particularmente coincido con Habermas, no se puede forzar de antemano a un ciudadano a que se exprese

\footnotetext{
${ }^{13}$ Habermas distingue entre "secular" y "secularista": "A diferencia de la actitud indiferente de una persona secular o no creyente que se comporta de una manera agnóstica frente a pretensiones de validez religiosas, los secularistas adoptan una actitud polémica frente a las doctrinas religiosas que, pese a sus pretensiones no fundamentables científicamente, gozan de una importancia pública. En la actualidad, el secularismo, suele estar basado en un naturalismo duro, es decir, fundamentado por la ciencia” (Habermas, 2015: 276).
} 
en un lenguaje que, o no siente propio, o no le es "suficiente"14. Pensemos en el siguiente caso, que no responde al ejemplo usual del ciudadano católico conservador frente a un Estado laico: la participación de un ciudadano argentino de origen wichi o mapuche en la esfera público-política. ¿Debería este ciudadano expresar sus demandas, por ejemplo, de propiedad de la tierra y del agua, fundamentales para su forma de vida, en un lenguaje "filtrado" de su particular cosmovisión? El carácter inclusivo del discurso compele a los interlocutores a oir y comprender sus argumentos, a fin de poder responder a la pretensión de validez elevada. Pero la pretensión de validez, en muchos casos, debe ser reconstruida como tal. En esto consiste fundamentalmente, a mi modo de ver, la traducción. En suma, por razones lógicas $y$ morales, debe favorecerse la integración en el discurso de quienes, por razones fácticas, culturales, políticas o económicas se encuentran fuera. Pero esto es algo diferente de lo que dice Habermas apenas unas líneas más debajo de la cita anterior: "Las tradiciones religiosas están provistas de una fuerza especial para articular intuiciones morales, sobre todo en atención a las formas sensibles de la convivencia humana. Este potencial, convierte al habla religiosa, cuando se trata de cuestiones políticas pertinentes, en un serio candidato para posibles contenidos de verdad, que pueden ser traducidos entonces desde el vocabulario de una comunidad religiosa determinada a un lenguaje universalmente accesible" (Habermas, 2006: 139). A mi juicio, aquí Habermas otorga una cierta prioridad o privilegio cognitivo a la religión que en mi opinión no tiene fundamento alguno. Sin embargo, sí es importante reconocer, como decía, el valor motivacional del discurso religioso, aunque, como todo valor, no esté exento de ambigüedad. A menudo, se interpreta esta especie de privilegio epistémico de la religión debido a su lugar en la historia de la cultura: de hecho, se argumenta, la filosofía no es más que una traducción de ese lenguaje. La traducción filosófica de la religión, y de los "filósofos traductores" es otra arista de la cuestión de la religión.

\section{c) La religión y su "traducción" por la filosofía}

Otra manera de situar lo religioso es, entonces, a través del valor que ha de otorgársele a las doctrinas religiosas en su relación con la filosofía. De algún

\footnotetext{
14 "La admisión en la esfera público-política de las manifestaciones religiosas que no han sido traducidas no sólo se justifica normativamente porque la estipulación rawlsiana no puede ser exigida razonablemente a aquellos creyentes que no pueden renunciar al uso político de razones presuntamente privadas o apolíticas sin poner en peligro su forma religiosa de vida" (Habermas, 2006: 138).
} 
modo, este es el aspecto más "clásico", en la medida que se trata de la relación entre estas dos figuras del espiritu. En general, tiene que ver también con el proceso de Ilustración, entendiendo a esta no sólo como un período particular de la historia europea, sino como el proceso de autodistanciamiento o de puesta en tela de juicio de las tradiciones o creencias religiosas. Ahora, si bien es innegable que las grandes cuestiones de la filosofía pueden ser retrotraídas genealógicamente a cuestiones religiosas, teológicas o metafísicas, uno puede preguntarse por qué habría de seguir indagándose en un contenido semántico que, al menos para un pensamiento posmetafísico, se encuentra ya superado; en palabras del ¿anterior? Habermas: "desde entonces [desde Hegel] carecemos de alternativas al pensamiento posmetafísico" (Habermas, 1988: 39). Ello no implica, por supuesto, negar el valor histórico-cultural y de autocomprensión que tal tarea supone, del mismo modo que tampoco implica una censura previa respecto del potencial semántico de los discursos de la mitología y la religión (en tanto capaces de aperturas de sentido, como el arte) y, en sentido estricto, de cualquier cosmovisión en general. Pero, así como no puede limitarse a priori, tampoco puede otorgársele a priori a la religión algún tipo de privilegio cognitivo.

Sin ánimo de dar una respuesta definitiva, creo que la propensión a buscar en las religiones o mitologías una fuente de sentido se debe, a mi juicio, a una suerte de "mito del origen", esto es, al prejuicio de que habría una suerte de cercanía con la sabiduría o la verdad en aquellas cosmovisiones que no han sido particularmente "contaminadas" por la modernidad ${ }^{15}$. Es difícil adscribir a Habermas esta intención, pero es sin duda uno de los supuestos que se desprenden del análisis de sus intervenciones. Sólo con fines ilustrativos, podemos mencionar el siguiente ejemplo ${ }^{16}$. En un texto reciente, de homenaje a Martin Buber, Habermas reflexiona sobre este estatus peculiar del filósofo o escritor religioso. Además de Buber, Habermas menciona a Kierkegaard, Royce, James, Bloch, Benjamin, Levinas y Derrida (Habermas, 2016: 37). ¿Cuál es la tarea que desempeña el filósofo o escritor religioso? En primer lugar, Habermas la sitúa en una dimensión temporal o histórica, es decir, desempeñan el papel de traductores dentro de un momento histórico o de transición epocal. Así, establece una analogía con "la metafísica griega bajo la

\footnotetext{
${ }^{15}$ Esto lo planteo indepedientemente de la cuestión de la imposibilidad de una lingüistificación completa de lo sagrado. Si hay algo de lo arcaico que se resiste es una cuestión abierta.

${ }^{16}$ La recepción por parte de Habermas de los filósofos-escritores religiosos y, consecuentemente, el análisis empírico reconstructivo de cómo la filosofía traduce el lenguaje religioso será el tema de un próximo trabajo. Si bien se trata de escritos ocasionales o que no tienen un carácter sistemático, valen como modelo para el tema de la traducción.
} 
tutela de la religión abrahámica tras la clausura de la Academia [...] Así, los filósofos debieron revelarse, en cierta medida, como escritores religiosos si querían traducir en conceptos filosóficos accesibles a la generalidad los contenidos semánticos básicos del articulado tesoro de una concreta tradición religiosa" (Habermas, 2016: 38). Del mismo modo, los filósofos escritores contemporáneos, como Buber, "retoman, bajo las condiciones propias de la Modernidad, una labor de traducción que puede entenderse en el modo de una ósmosis que pasa desapercibida..." (Habermas, 2016: 37). En segundo lugar, en consecuencia, estos pensadores enriquecieron la esfera pública gracias a que "filtraron filosóficamente los contenidos de la experiencia religiosa y eliminaron de ellos el carácter exclusivo que viene dado por su origen en una comunidad religiosa" (Habermas, 2016: 38). Es decir que los "filósofos traductores" desempeñaron y desempeñan una doble tarea: histórica y político-pública, en otras palabras, se trata de una tarea de Ilustración. Si esta tarea concluirá algún día no puede saberse, y en eso radica el cambio de actitud del secularismo, pero es claro, a mi juicio, que Habermas, si bien no profetiza el fin de la religión sí de algún modo promueve su traducción filosófica, esto es, argumentativa y pública. A mi modo de ver, el presunto giro religioso de Habermas consiste, en este punto, en inyectar una dosis de falibilismo en el triunfalismo secularista, pero la religión no deja de ser, en cierto modo, algo "superable" por la filosofía. En este sentido, por ejemplo, Michelini ha planteado una objeción importante: no todos los contenidos religiosos son necesariamente traducibles, sino sólo aquellos de importancia pública o con pretensiones normativas, y querer traducir toda la religión implicaría la desaparición misma de la religión. A ello podría responderse, sin embargo, que lo que determina a la religión como tal, más que sus contenidos, es el culto o sus prácticas rituales. Pero esta es una cuestión que dejo abierta.

De esta multiplicidad de aristas del tema de la religión ${ }^{17}$, tal como adelanté en la introducción, quisiera concentrarme en un punto en particular, a saber: el problema

\footnotetext{
${ }^{17}$ Por razones de espacio, lamentablemente, debo dejar sin desarrollar dos de estas dimensiones. La primera, la relación con el mito, el ritual y lo que Habermas denomina "lingüistificación de lo sagrado”, es el aspecto, desde mi punto de vista, más relevante y más denso teóricamente, y al que aún no se le ha dedicado toda la atención que merece. En otros términos, se trata de lo programáticamente más prometedor de los últimos trabajos filosóficos de Habermas (influenciado en gran medida por los estudios de Tomasello sobre el lenguaje y la comunicación en primates y niños). En síntesis, Habermas ahora rechaza que la acción comunicativa pueda lingüistificar lo sagrado de manera completa, ver Rosati (2014). El segundo aspecto es el que a mi modo de ver inicia este "giro" de Habermas hacia lo religioso, y es su ensayo sobre la eugenesia liberal.
} 
de la traducción del contenido semántico del discurso religioso al ámbito público. $\mathrm{Al}$ contrario de quienes piensan que este es un punto ya discutido y superado o que no tiene mayor potencial, creo que se trata de un aspecto de la discusión general sobre la religión en el espacio público sobre el que todavía queda por analizar.

\section{3. ¿QUÉ PUEDE SIGNIFICAR “TRADUCIR” UN ARGUMENTO?}

En la introducción afirmé que es posible entender la traducción, en este marco, en dos sentidos: como desencapsulamiento y como cambio de género discursivo. Esta hipótesis tiene un carácter eminentemente exploratorio, simplemente intenta aclarar qué puede querer decir "traducir" una razón. Además de los propios textos de Habermas, y como veremos más adelante, de Ferry, apelo a la hermenéutica trascendental a la que me he referido en otros trabajos.

\section{a) Traducción como "desencapsulamiento"}

El debate en torno a la secularización y la modernización social abre, entonces, dos cuestiones para la filosofía y su relación con la religión: por un lado, el vínculo propiamente dicho entre filosofía y religión, y por otro, el problema filosófico-político en torno al rol de las comunidades religiosas dentro de un Estado entendido como secular (Habermas, 2015: 13, 135). Sin embargo, habría una relación entre ambas cuestiones — la de la relación filosofía/religión y la cuestión político normativa relativa al rol de la religión en el ámbito público- ya desde el momento que los ciudadanos tienen que presentar argumentos o razones para apoyar o criticar sus posiciones políticas, pero muchas de esas razones se fundan en convicciones de tipo religiosas y, aunque sea una exigencia de los Estados laicos, es poco creíble pensar que un ciudadano religioso simplemente pueda dejar sus convicciones - como quien deja un saco o los zapatos- a la puerta de ingreso a la esfera público-política. Pero el problema que presentan las contribuciones religiosas es que vienen formuladas en un lenguaje que, en principio, se encuentra "encapsulado"18. Con ello quiero decir que el contenido material de las razones

\footnotetext{
${ }^{18}$ En Nachmetaphysichen Denken II, Habermas realiza una autocrítica respecto de la lingüistización de lo sagrado, en ese marco, afirma: "A la luz de la diferenciación entre comunicación cotidiana y extracotidiana, me represento la lingüistización de lo sagrado en la actualidad de otra manera. Hubo que liberar los contenidos normativos de su encapsulamiento ritual y luego trasladarlos a la semántica del lenguaje cotidiano" (Habermas, 2015: 16). En tanto que a la filosofía, según Habermas, se le presenta la tarea de continuar con la lingüistización de lo sagrado, esto es, "descubrir en las tradiciones religiosas los potenciales semánticos todavía no saldados, y
} 
en tanto argumentos se encuentra, prima facie, formulado en un lenguaje aceptado por y dirigido a una determinada comunidad que, por otra parte, establece como criterio de pertenencia la aceptación de un número determinado de "verdades", en principio resignificables, pero no cuestionables. Habermas propone una "cláusula de traducción" a dichas comunidades, la cual consiste en traducir sus respectivas contribuciones a un "lenguaje de acceso universal, antes de que su contenido pueda encontrar entrada en las deliberaciones de aquellas instituciones políticas que toman decisiones jurídicamente vinculantes" (Habermas, 2015: 136). Con ello se alcanzaría, por así decir, una salomónica solución política, que, como el mismo Habermas sostiene, para algunos es demasiado y para otros demasiado poco. Las comunidades religiosas, según Habermas, no los individuos por separado, deberían entonces secularizar sus razones, esto es, formularlas en un lenguaje "público". En este sentido, según entiendo, las comunidades religiosas deberían institucionalizar comunidades de traducción para que sus pretensiones de validez sean traducidas a un lenguaje secular a fin de obtener el pasaporte de ingreso a la esfera público-política.

Creo que esta versión del problema adolece de una serie de dificultades. En primer lugar, una de las principales dificultades que se presentan con las llamadas contribuciones religiosas no es primeramente el lenguaje en el que están basadas sino el carácter dogmático de dichas contribuciones, esto es, la falta de disponibilidad para reconocer que el sentido, la verdad o lo correcto puede no ser necesariamente el que se corresponde a su posición inicial. En otras palabras, el problema consiste en la pretensión de infalibilidad de sus expresiones, que reclaman ciertas instituciones religiosas. En algunos debates políticos, por ejemplo, es notable incluso cómo se apela a una especie de "secularismo estratégico", es decir, en la mera reformulación del lenguaje teológico a través del lenguaje del derecho o de la ciencia con fines no de elevar una pretensión de validez normativa "genuina" sino de presentar una posición ya asumida de antemano como absoluta e infalible de forma más atractiva para oídos no religiosos o científicos. Un ejemplo práctico de esto es, como ya mencioné antes, la apelación que hacen los llamados grupos provida que, utilizando el lenguaje de la genética o de la biología, recubren sus pretensiones normativas basadas en la voluntad de Dios para oponerse a la legalización

traducirlos con sus propios medios en un lenguaje universal y accesible, más allá de esas determinadas comunidades religiosas, para alimentar de esta manera el juego discursivo de los argumentos públicos" (Habermas, 2015: 19), la traducción consistiría en ese liberar el contenido semántico encapsulado, de allí entonces, la idea de "desencapsulamiento". 
del aborto. Ahora bien, esta reformulación, ¿no es precisamente un ejemplo de traducción del lenguaje religioso al lenguaje secular? ¿Hasta qué punto una razón es traducida o reformulada y hasta qué punto una razón es "disfrazada"? En esta imposibilidad de determinar cuándo una traducción es genuina, cuándo es falsa o incompleta se basa una de las principales razones de escepticismo respecto de la participación genuina de la religión en el ámbito público. Con lo cual, la cuestión de la traducción es mucho más relevante de lo que podría pensarse, porque si efectivamente no es posible traducir razones religiosas entonces en sentido estricto tales razones no pueden participar de la esfera pública.

Ahora bien, la cuestión del lenguaje semánticamente encapsulado, por así decir, no es cuestión que atañe sólo a los discursos religiosos. A mi modo de ver, cualquier "discurso" se encuentra cargado semánticamente por entramados conceptuales o matrices de interpretación (Wellmer, 1994). En una entrevista, Habermas aclara su crítica al modelo laicista de separación entre Estado e Iglesia afirmando que: "lo que debe salvaguardarse es que las decisiones del poder legislativo, del ejecutivo y del judicial estén no sólo formuladas en un lenguaje universalmente accesible sino también que estén justificadas sobre la base de razones aceptables por todos universalmente" (Habermas, 2015: 101-102). Pero en la esfera públicopolítica informal no se puede trazar un límite entre lo secular permitido y lo religioso prohibido. Un ejemplo recurrente en estas discusiones (en Aguirre, Lafont, Walhof) es el caso del matrimonio entre personas del mismo sexo. Por ejemplo, la Iglesia Católica puede afirmar que se trata de una acción contraria a la voluntad de Dios (y lo sigue sosteniendo de hecho, aun cuando por ejemplo en Argentina sea legal desde hace seis años), o también se puede esgrimir el argumento de que se trata de una acción contraria al "orden natural". Claramente el argumento que apela a la "voluntad de Dios" puede ser objetado porque es exclusivista, sólo tiene sentido para quienes ya creen en Dios y en ese Dios. En cambio, el concepto de orden natural es más ambiguo, pero, ¿se trata de un argumento secular? ¿Hay aquí una traducción secular del lenguaje religioso? ¿O se trata más bien de un argumento completamente diferente?

Por lo tanto, creo que es importante definir qué se entiende por lenguaje secular. A mi modo de ver no se trata de un lenguaje en sentido estricto sino de un género, esto es, la argumentación. De lo contrario, se corre el peligro de identificar el lenguaje secular con una forma de vida concreta, a saber, la que corresponde con las sociedades modernas occidentales, y legítimamente se podría reclamar que las religiones se ven forzadas a hablar en un lenguaje que no les es propio debido a una especie de "coacción” cultural (Michelini, 2015): de nuevo, la coacción que 
ejercerían las sociedades modernas eurocéntricas que separan Estado e Iglesia, etc. Ahora bien, aun cuando consideremos el lenguaje secular como un tipo de género discursivo, la realización efectiva de ese lenguaje se realiza en un tipo de lenguaje concreto, con sentidos sedimentados a lo largo de siglos. El lenguaje, entonces, no puede ser neutro ni a-cosmovisivo, por eso, a mi modo de ver, tiene un peso específico mucho mayor que el que, al menos Habermas, le otorga en relación con la posibilidad del entendimiento (Verständigung). Sin embargo, no tiene la religión un lugar "especial" en este aspecto, y si lo tiene, no se debe, a mi juicio, a su tipo de lenguaje, sino al valor que se le otorga y al tipo de poder que está en condiciones de ejercer en el circuito general de la comunicación política. ${ }^{19}$

\section{b) Religión: actitud pragmática y actitud semántica}

En un artículo reciente, Jean-Marc Ferry, aclara esta distinción entre la dimensión pragmática y semántica de las condiciones de acceso de la religión a la esfera pública $^{20}$. En primer lugar, Ferry parte, como Habermas, del reconocimiento del sistema político (en su caso la Unión Europea) de la importancia de la religión. Si bien en el proceso de diferenciación social de la modernidad la religión ha pasado a ser una esfera más, y opcional, en una sociedad "postsecular" se busca superar el presunto carácter privado de la religión: "Una sociedad postsecular es capaz de ofrecer, sobre una base igualitaria, un marco apropiado para una exposición pública de las convicciones sometida a la prueba de contra-experiencias y contraargumentos" (Ferry, 2016: 27). Ferry prefiere el término "secularización interna" o "segunda", en la medida que entiende que no están puestos en tela de juicio los objetivos de la laicidad. El punto es que el postsecularismo apuntaría no a la "disminución de la diversidad étnica y religiosa" sino al "reconocimiento sustancial de convicciones", "sugiere un levantamiento del velo de ignorancia puesto sobre las pertenencias y las convicciones" (Ferry, 2016: 28). Ahora bien, esta ampliación de la arena deliberativa supone también criterios de admisibilidad de los argumentos con fines normativos. En ese sentido, por ejemplo, los argumentos comprendidos

\footnotetext{
${ }^{19}$ Habermas divide el circuito de la comunicación política en tres niveles: los discursos institucionalizados, que corresponde básicamente a la esfera del Estado, la comunicación de masas basada en los medios de comunicación y la comunicación cotidiana de la sociedad civil. En el segundo nivel, las elites impulsan la comunicación mediática, entre ellos debe contarse a las iglesias. Ver, Habermas, 2009: 158 ss.

${ }^{20} \mathrm{El}$ texto español utilizado en este trabajo corresponde al capítulo II y parte del III de su libro La Raison et la Foi.
} 
bajo la cláusula "Dios dice que..." ya no son admisibles. Ferry aboga entonces por una perlaboración de la política con la religión. No me ocuparé aquí de la dimensión política, aunque si es digno de mencionar su crítica al modelo democrático liberal y a su idea de consenso como solapamiento, en la medida que allí se corre "el peligro de vaciamiento de la base prepolítica de la solidaridad cívica" (Ferry, 2016: 36). Esto implica el traspaso hacia un modelo de "consenso por confrontación" (a diferencia del consenso por "solapamiento" de Rawls), lo cual supone una ampliación a "registros discursivos que no se reducen al estilo de la argumentación jurídica" (Ferry, 2016: 34).

Pero quisiera concentrarme aquí en las condiciones o "cambios de actitud" que deberían asumir las religiones para implicarse en los procesos de la razón pública, cambio al que las religiones han sido "invitadas" desde el sistema político mismo. Este cambio supone dos dimensiones, una pragmática y la otra semántica (Ferry, 2016: 38 ss.). El cambio pragmático se refiere a la interiorización de las religiones de los principios de una sociedad abierta: falibilismo, criticismo y perspectivismo. Este cambio de actitud es mucho más complejo, a mi juicio, de lo que muchos autores sensibles a la religión suponen, pues no sólo consiste en expresarse públicamente acerca de los asuntos públicos — como bien señala Ferry, algo a lo que las propias Iglesias ya se autorizan-. De hecho, es casi una constante que, frente a debates públicos, como mencionábamos al comienzo, las diferentes confesiones se expresen públicamente, generalmente en contra: es el caso del divorcio, el matrimonio homosexual, el uso de anticonceptivos, el aborto, y un largo etcétera. Sin embargo, asumir el principio del falibilismo supone precisamente reconocer la posibilidad de estar en el error, algo que generalmente las religiones no asumen por basarse en supuestas verdades reveladas. Por criticismo entiende Ferry, la disposición a ofrecer justificaciones y a admitir refutaciones, "sobre la base de exámenes imparciales". Por perspectivismo, finalmente, la idea de que "el contenido de cualquier proposición sólo cobra sentido en función del punto de vista desde el cual se enuncia" (Ferry, 2016: 38). Estas tres actitudes implican un comportamiento reflexivo que parte de la base de que es posible distinguir entre certeza o convicciones y verdad. Pero, en muchos casos, esa conciencia reflexiva no está presente en las religiones, que parten de certezas consideradas como verdades. Ferry conecta este "ethos falibilista-perspectivista-criticista" con los presupuestos contrafácticos descriptos por la ética discursiva. En definitiva, quien no asume tales presupuestos no participa en una "discusión verdadera", dice Ferry. Precisamente, en muchos casos podemos poner en duda si determinadas religiones efectivamente quieren entrar en el discurso y someterse a la fuerza de los mejores 
argumentos. Pero pongamos entre paréntesis el escepticismo y concedamos este punto. Además de este cambio de actitud pragmático se requiere de un segundo, de tipo semántico.

Según Ferry, el cambio semántico supone que las religiones "pongan las cartas sobre la mesa y se esfuercen por explicitar, más allá de un «Dios dice que...» las bases de las convicciones y de las doctrinas de su toma de posición..." (Ferry, 2016: 40). Básicamente, se trata de un proceso de traducción, en total coincidencia con lo propuesto por Habermas ${ }^{21}$. Sólo que, en el caso de Ferry, parece tratarse de una traducción realizada desde la propia comunidad religiosa hacia la razón pública, cuando Habermas habla más bien de una tarea cooperativa entre ciudadanos religiosos y seculares. (Más adelante volveré sobre este punto). En este texto, Ferry presenta tres ejemplos de traducción del lenguaje religioso a uno secular: la creación del hombre a "imagen y semejanza" de Dios puede ser traducido como el carácter sagrado y diferenciado de la persona humana; la unicidad de Dios y de que seamos Sus hijos se puede traducir en la hermandad y consecuentemente igualdad en dignidad de todos los hombres; o el argumento de la creación del mundo y de la naturaleza como una forma de entender la naturaleza no como una propiedad del hombre sino como objeto de cuidado. Estos ejemplos, entre muchos otros, mostrarían entonces, según este autor, que la traducción no sólo es un requisito necesario, sino que además es posible. Incluso Ferry llega a afirmar que "todos los géneros semióticos pueden ser traducidos al lenguaje común, pues todas las intuiciones se benefician de la presunción de comunicabilidad" (Ferry, 2016: 41), con lo cual no sólo la religión formal o estándar, por así decir, podría ser traducida, sino también el mito o cualquier otra construcción narrativa. Esta mediación "teológica" (semiótica) genera contenidos que luego la razón pública podría o no retener, mediante la mediación jurídica. (Sin embargo, no quisiera detenerme en este aspecto, pues creo que es el menos problemático, y en el que, al fin y al cabo, todos los autores que estamos analizando coinciden: el laicismo).

De acuerdo con Ferry, el problema de la traducción es un problema gramatical (noción que el autor desarrolla en Grammaires de l'intelligence). La diferencia del lenguaje religioso con el secular radica en su diferente gramaticalidad, la religión

\footnotetext{
${ }^{21}$ Esto no lo ve así Graziano Lingua, quien pone el acento en la traducción en el segundo sentido, que aquí llamo "cambio de género discursivo". Lamentablemente, más allá de lo sugerente que representa la perspectiva de este autor, sobre todo a partir de Ricoeur, no brinda ejemplos claros de cómo se lleva a cabo efectivamente este tipo de traducción, al menos en el texto que he tenido en cuenta aquí: "Dépasser l'ex-communication politique du religieux" (Landenne, 2014: 74 ss.).
} 
se encuentra más ligada a los registros narrativos e interpretativos mientras que la gramática jurídica apela a la argumentación (Ferry, 2016: 51). En cierto sentido, en esto consiste para Ferry el "paso" del mithos al logos, y, sin identificar igualmente mito y religión, sí lo que importa aquí es que la tarea del logos es la reconstrucción: "Sin la mediación del registro reconstructivo, el retorno sobre lo narrativo conlleva la temeraria aspiración a un reencantamiento del mundo" (Ferry, 2016: 52).

\section{c) Traducción como cambio de género discursivo}

La traducción entonces puede consistir en un proceso mediante el cual se reformula una determinada posición (pretensión de validez) en un lenguaje universalmente accesible. Pero como vimos, este puede ser el caso para traducciones de las del tipo de Ferry: "dado que hemos sido creado a imagen y semejanza de Dios, todos somos iguales", a, por ejemplo, "en tanto sujetos racionales, todos somos iguales". Como se puede apreciar, aquí no se juzga sobre el argumento en sí, esto es, si la afirmación es correcta, sino sobre su formulación. Lo discutible, como vimos, es si la segunda formulación efectivamente mantiene el sentido de la primera. Sin embargo, podríamos decir que se trata de dos argumentos en sentido propio, más allá del contenido semántico. Otro problema, más complejo a mi juicio, es cuando se trata de traducir lenguajes o géneros no argumentativos o para-argumentativos al discurso. En los textos considerados sagrados y canónicos, al menos por las "grandes religiones mundiales", encontramos abundante material: parábolas, sermones, exhortaciones, poesías. Algunos sostienen que el discurso religioso es difícilmente traducible o que directamente es intraducible (Lara, 2013: 155). Sobre esto creo que pueden decirse dos cosas. Si es fácil o difícil, lo que sea que signifique eso, no es el punto de discusión, el punto de discusión es cómo podría participar una pretensión normativa, por ejemplo, en la esfera pública, si no se formula o no se puede comprender como pretensión de validez. Por otro lado, la historia está repleta de intervenciones bastante claras de las religiones en la esfera pública. Cuando por ejemplo el Cardenal Bergoglio, ahora Papa Francisco, se refirió al matrimonio entre personas del mismo sexo como algo "demoníaco", dejaba bastante en claro lo que piensa la Iglesia Católica sobre la homosexualidad. Tal vez esta condena religiosa pueda ser traducida a un lenguaje secular con pretensiones tales como "las relaciones sexuales y consecuentemente toda forma de unión bajo la figura del matrimonio, son incorrectas y el Estado no deberían alentarlos", "la homosexualidad es antinatural" o "la homosexualidad es una enfermedad", o argumentos por el estilo. Entonces, no se trata de que "la" religión sea traducida (Michelini, 2015), sino sus pretensiones normativas: 
de nuevo, la oposición de la Iglesia Católica a la legalización del aborto ni es un "misterio insondable" ni es sólo una recomendación a sus fieles, se trata de una posición que tiene pretensión de incidir en la esfera política, esto es, impedir que exista tal ley para todas las mujeres — sean o no católicas_- que los Magistrados no puedan impartir justicia bajo su imperio y que las instituciones y funcionarios del Estado no se rijan por ella. Ahora bien, realizada esta aclaración, también hay que conceder que ciertas intuiciones morales, formuladas en un género no discursivo, puedan querer ingresar a la esfera pública y que, por las razones que fuera, el o la interlocutora se ve imposibilitado de formularlo como un argumento público. En tal caso, los interlocutores, que quieren entenderse y por lo tanto encontrar una base común de significado, deben desempeñar un esfuerzo de traducción para la comprensión mutua de sus argumentos. Pero, como vimos, una cosa es traducir entre una misma "gramática" y otra entre diferentes "gramáticas" (Ferry, 2016). Para este segundo caso, lamentablemente casi no tenemos indicaciones en los textos de Habermas, excepto por una vaga nota a pie de página respecto del uso comunicativo de las parábolas bíblicas en la teología de Edmund Arens. Sin embargo, creo que esta segunda dimensión de la traducción nos lleva más allá de Habermas. Tal vez la interpretación de Ferry en la línea de la tradición de Ricoeur pueda ofrecer una perspectiva más amplia o clarificadora. Pero esta es una cuestión que quedará abierta a futuros trabajos.

\section{A MODO DE CONCLUSIÓN}

En este trabajo he intentado defender, en el aspecto político, que las religiones no ocupan ningún lugar "especial" o "privilegiado" en la esfera público-política, en cuanto a su presunta capacidad de dotación de sentido y motivación (no al menos en cuanto a otras expresiones del espíritu, como la literatura o el arte); más aún, dentro del esquema de la comunicación público-política las religiones ocupan el mismo lugar que los grupos de presión, abogados de intereses generales o formadores de opinión en general. Si bien coincido en que es necesario un filtro institucional al lenguaje religioso, el cual consiste en un proceso de traducción, no considero que esta sea una tarea especial o específica que supuestamente deberían desempeñar los ciudadanos seculares, pues en cierto sentido, en un discurso todos somos potenciales traductores. De hecho, la distinción entre ciudadanos seculares y ciudadanos religiosos, además de problemática desde un punto de vista empírico, es irrelevante para el problema de la conformación de la voluntad política en un Estado de Derecho. Sean cuales fueran las matrices de interpretación (y con esto quiero ir más allá de la siempre engorrosa cuestión de delimitar qué es religión, 
cosmovisión, espiritualidad, o "doctrina comprehensiva") de los participantes en un discurso, los argumentos a favor o en contra de determinadas acciones deben ser presentados públicamente, formulados de una manera comprensible para todos y cuya justificación debe satisfacer a todos, esto, además con el compromiso contrafáctico de reformulación de las razones a requerimiento del resto de los interlocutores. El aspecto de la reformulación (que no implica un cambio de razones) tal vez no ha sido tan tenido en cuenta en la ética discursiva. Al menos en Habermas, la traducción cumple la función de construir una base de sentido general, necesaria para poder debatir públicamente. Sin un significado intersubjetivamente válido no se puede dirimir ninguna pretensión de validez.

He intentado sostener, además, que quienes ingresan en un discurso se presuponen recíprocamente también competencias hermenéuticas tales como las de la interpretación del contenido proposicional de sus actos de habla o eventualmente de "traducción" de sus manifestaciones. Hasta el momento, creo que, en este marco, puede entenderse por traducción dos cosas, no contrapuestas. En primer lugar, la "apertura" de un lenguaje, esto es, la reformulación de determinados enunciados en un lenguaje que vaya más allá de los destinatarios iniciales o de la comunidad de pertenencia. Por ejemplo, la exhortación a comportarse como "hermanos" (en un lenguaje religioso que adscribe a "Dios" la paternidad) puede ser reformulado en términos de comportarse como "iguales". En segundo lugar, la traducción puede consistir en la reformulación de géneros no discursivos, como las parábolas o cualquier otro tipo de lenguaje narrativo en general, pero con pretensiones normativas, en actos de habla argumentativos. Sin embargo, si bien el discurso religioso no es un caso especial, la discusión sobre su eventual traducción al lenguaje secular sí permite avanzar en la cuestión mencionada: el problema de la traducción del contenido semántico del discurso religioso abre el campo problemático de la comprensibilidad en general como un elemento a tener en cuenta en la puesta en práctica o institucionalización del discurso.

Gonzalo Scivoletto

CONICET (Argentina) scivolettog@gmail.com 


\section{BIBLIOGRAFÍA}

Aguirre, J. (2012), "Jürgen Habermas y la religión en la esfera pública”, en Ideas y valores, lxi, 148, Bogotá, 59-78.

- (2015). Hacia un sentido democrático de "lo político": Jürgen Habermas y el rol de la religión en la esfera pública. Revista de Antropología y Sociología: Virajes, 17 (1), 23-44.

APEL, K.-O. (1997), "Plurality of Good? The Problem of Affirmative Tolerance in a Multicultural Society from an Ethical Point of View", Ratio Juris 10, 2: 199-212.

- (1998), Auseinandersetzungen in Erprobung des transz̨endentalpragmatischen Ansatzes, Frankfurt am Main: Suhrkamp.

- (2001), The Response of Discourse Ethics to the Moral Challenge of the Human Situation as Such and Specially Today, Leuven: Peeters.

- (2008), "The hermeneutics of Being versus transcendental hermeneutics or transcendental pragmatics", en The Routledge Companion to Twenty Century Philosophy, London and New York: Routledge, 736-783.

- (2009), Semiótica filosófica, Buenos Aires: Prometeo Libros.

- (2013), Paradigmas de filosofía primera, Buenos Aires: Prometeo Libros.

ARFI, B. (2015), "Habermas and the aporia of translating religion in democracy", en European Journal of Social Theory, 18 (4), 489-506.

BÖHLER, D. (1982), "Transzendentalpragmatik und kritische Moral. Über die Möglichkeit und die moralische Bedeutung einer Selbstaufklärung der Vernunft", en Kuhlmann, W. yBöhler,D. (eds.). Kommunikation undReflexion. ZurDiskussionderTranszendentalpragmatik Antworten auf Karl-Otto Apel, Frankfurt: Suhrkamp.

BuRCKHART, H. (2000), Nichthintergehbarkeit und Unverzichtbarkeit einer diskursethischen Begründung von Moral, en Burckhart, H. y Reich, K. (eds.), Begründung von Moral: Diskursethik versus Konstruktivismus, Würzburg: Königshausen und Neumann.

CoOKE, M., "Translating truth", Disponible en http:/ / www.resetdoc.org/story/00000022223 (última consulta 30-10-2015).

- (2006), "Salvaging and secularizing the semantic contents of religion: the limitations of Habermas's postmetaphysical proposal", en International Journal for Philosophy of Religion, 60 (1/3), 187-207.

FERRY, J.-M. (2016), "La religión in foro publico", en Romerales, E. y Zazo, E. (coords.), Religiones en el espacio público, Barcelona: Gedisa.

GÓMEZ RINCÓN, C. M. (2015). La transformación postsecular de la relación entre religión y racionalidad. Ideas y Valores, 64 (157), 71-90.

Habermas, J. (1988), Pensamiento posmetafísico, Madrid: Trotta.

- (1996), Textos y contextos, Barcelona: Ariel.

- (2002), El futuro de la naturaleza bumana, Barcelona: Paidós.

- (2006), Entre naturalismo y religión, Barcelona: Paidós.

- (2009), ;Ay, Europa!, Madrid: Trotta. 
Habermas, J. (2010), Teoría de la acción comunicativa, Madrid: Trotta.

- (2015), Mundo de la vida, política y religión, Madrid: Trotta.

- (2016), En la espiral de la democracia, Madrid: Trotta.

HARrington, A. (2007), "Habermas and the 'Post-Secular Society", en European Journal of Social Theory 10(4): 543-560.

LAFOnT, C. (2002), The Linguistic Turn in Hermeneutic Philsophy, MIT Press.

- (2009), "Religion and the public sphere. What are the deliberative obligations of democratic citizenship? Philosophy \& Social Criticism, 35, 1-2, 127-150.

— (2010), "Dos argumentos transcendentales contra la tesis de la inconmensurabilidad", en Giusti, M. (ed.), Tolerancia, Conferencias plenarias del XV Congreso Interamericano de Filosofía y el II Congreso Iberoamericano de Filosofía, Madrid.

LANDENnE, Q. (dir.) (2014), La philosophie reconstructive en discussions. Dialogues avec Jean-Marc Ferry, LE BORD DE L'EAU.

LARA, M. (2013), The Disclosure of Politics. Struggles over the Semantics of Secularization, New York: Columbia University Press.

LÓPEZ DE LIZAGA, J. (2011), "Verdades religiosas, política laica: Habermas sobre la religión en la esfera pública”, en Franzé, J., Política y verdad, Madrid: Plaza y Valdés, 175-208.

MARDOnES, J. (1998), El discurso religioso de la modernidad. Habermas y la religión, Barcelona: Anthropos.

MENDIETA, E. (2001), “Introducción. La lingüistificación de lo sagrado como catalizador de la modernidad", en Habermas, J., Israel o Atenas, Madrid: Trotta, 11-50.

- y Vanantwerpen, J. (eds.) (2011), Elpoder de la religión en la esfera pública, Madrid: Trotta.

MiCHELINI, D. (2015), “Creyentes y no creyentes en una sociedad democrática. La religión en el pensamiento actual de Jürgen Habermas”, en Invenio, 18 (35), 11-27.

MuÑoz FonNEGRA, S. (2013), "La función público-política de la religión. Reflexiones sobre la controvertida interpretación de Habermas", en Astrolabio. Revista internacional de filosofía, 15, 32-41.

PronO, S. (2016), "Política y religión en Habermas. Reflexiones críticas desde la teoría del discurso", en Estudios de Filosofía Práctica e Historia de las Ideas, 18, Disponible en http://qellqasqa.com.ar/ojs/index.php/estudios/article/view/137 (Última consulta: 6 de octubre de 2016).

Rosati, M. (2014), "The Archaic and Us. Ritual, Myth, the Sacred and Modernity", en Reset DOC, disponible en: http://www.resetdoc.org/story/00000022339 (Última consulta: 6 de octubre de 2016).

TAYLOR, C. (2015), La era secular, Tomo II, Barcelona: Gedisa.

UNGUREANU, C. (2013), "Razón pública, religión y traducción: prospectivas y límites del postsecularismo de Habermas", en Revista Española de Ciencia Política, 32, 183-201.

Wellmer, A. (1994), Ética y diálogo. Elementos del juicio moral en Kant y la ética del discurso, Barcelona: Anthropos. 